IFIC/14-37

\title{
A model-independent fit to Planck and BICEP2 data
}

\author{
Laura Barranco, ${ }^{1}$ Lotfi Boubekeur, ${ }^{1,2}$ and Olga Mena ${ }^{1}$ \\ ${ }^{1}$ Instituto de Física Corpuscular (IFIC), CSIC-Universitat de Valencia, \\ Apartado de Correos 22085, E-46071, Spain. \\ ${ }^{2}$ Laboratoire de physique mathematique et subatomique (LPMS) \\ Universite de Constantine I, Constantine 25000, Algeria.
}

\begin{abstract}
Inflation is the leading theory to describe elegantly the initial conditions that led to structure formation in our universe. In this paper, we present a novel phenomenological fit to the Planck, WMAP polarisation (WP) and the BICEP2 datasets using an alternative parameterisation. Instead of starting from inflationary potentials and computing the inflationary observables, we use a phenomenological parameterisation due to Mukhanov, describing inflation by an effective equationof-state, in terms of the number of e-folds and two phenomenological parameters $\alpha$ and $\beta$. Within such a parametrisation, which captures the different inflationary models in a model-independent way, the values of the scalar spectral index $n_{s}$, its running and the tensor-to-scalar ratio $r$ are predicted, given a set of parameters $(\alpha, \beta)$. We perform a Markov Chain Monte Carlo analysis of these parameters, and we show that the combined analysis of Planck and WP data favours the Starobinsky and Higgs inflation scenarios. Assuming that the BICEP2 signal is not entirely due to foregrounds, the addition of this last data set prefers instead the $\phi^{2}$ chaotic models. The constraint we get from Planck and WP data alone on the derived tensor-to-scalar ratio is $r<0.18$ at $95 \%$ CL, value which is consistent with the one quoted from the BICEP2 collaboration analysis, $r=0.16_{-0.05}^{+0-06}$, after foreground subtraction. This is not necessarily at odds with the $2 \sigma$ tension found between Planck and BICEP2 measurements when analysing data in terms of the usual $n_{s}$ and $r$ parameters, given that the parameterisation used here includes, implicitly, a running spectral index.
\end{abstract}

PACS numbers: 98.70.Vc, 98.80.Cq, 98.80.Bp

\section{I. INTRODUCTION}

The recent claimed discovery of primordial $B$-modes by the BICEP2 collaboration $[1,2]$ has spurred a lot of interest in the cosmology community. One of the main topics of discussion is the tension between the BICEP2 results and the previous ones. In particular, this measurement corresponds to a tensor-to-scalar ratio of* $r=0.2_{-0.05}^{+0.07}$, while the Planck TT data (combined with WP data, high- $\ell$ CMB measurements and without running of the scalar spectral index) [3, 4] gives $r<0.11$ at $95 \%$ CL. As argued in [1], allowing for a running of the scalar spectral index makes the two datasets compatible at the one-sigma level. On the other hand, the largefield slow-roll models are able to explain successfully the BICEP2 data, however they predict negligible running, which, indeed, has not been seen in any previous observation like e.g. Planck. This by itself suggests a non-trivial departure from the simple single-field slow-roll inflation paradigm. Plenty of effort has been devoted in the literature to reconcile BICEP2 and Planck observations, either by modifications of the inflationary sector [5-8] and/or of the standard cosmological scenario, as, for instance, extensions to the neutrino sector [9-14]. Implications of the BICEP2 results in terms of the usual inflationary

\footnotetext{
* This figure is obtained without subtracting polarised dust foregrounds, though the signal seen by BICEP 2 outweigh any known foreground. Using the best available foreground template shifts the measured value to $r=0.16_{-0.05}^{+0.06}$.
}

parameters have also been extensively explored [15-17]. In this work, we will look at this issue with a different perspective; we shall use an alternative parameterisation to fit both Planck and BICEP2 observations. There are two aspects of this discrepancy that are worth pursuing. The first one is purely experimental/observational and implies a re-assessing of all the systematic errors and possible unaccounted-for foregrounds (see [18] for a recent analysis in this direction). Despite the tremendous and impressive work done by the BICEP2 collaboration, this step is mandatory before drawing any definitive conclusion about the cosmological origin of this signal. For a roadmap of this program, see e.g. [19]. In the following, we will be assuming that the BICEP2 signal is primordial, although the novel phenomenological approach presented here can be applied to fit any cosmological data. The second aspect is theoretical, and it addresses the crucial question: did our universe suffer a quasi de Sitter expansion phase driven by the potential energy of a scalar field (the inflaton)? if yes, then, among the variety of available inflationary scenarios, which one describes better the observations? and, what are the physical implications of such a scenario? In treating this last question, it is customary to use single-field slow-roll models as benchmark scenarios against which the temperature anisotropies observational data are tested. This is justified by the simplicity of these models when it comes to compute their predictions. Given a simple potential $V(\phi)$, where $\phi$ is the canonically-normalized inflaton field, one can compute easily the observational predictions in terms of the slow-roll parameters $\epsilon$ and $\eta$ defined 
$\mathrm{as}^{\dagger}$

$$
\epsilon \equiv \frac{1}{2} M_{P}^{2}\left(V^{\prime} / V\right)^{2} \text { and } \eta \equiv M_{P}^{2} V^{\prime \prime} / V ;
$$

where the primes denote derivatives with respect to $\phi$, i.e. $V^{\prime} \equiv d V / d \phi$ and so on. During slow-roll, these parameters are small i.e. $\epsilon,|\eta| \ll 1$, and the energy density of the universe is given approximately by the potential $V \simeq 3 M_{P}^{2} H^{2}$, where $H$ is the Hubble expansion rate during inflation. At leading order in slow-roll, the basic observables: the tensor-to-scalar ratio $r$ and the spectral index $n_{s}$, are given by

$$
r=16 \epsilon_{*} \text { and } n_{s}=1+2 \eta_{*}-6 \epsilon_{*},
$$

where the $\operatorname{subscript} *$ is to remind that quantities are evaluated at horizon exit. These quantities are usually the basic ones used when testing models against observations. Each potential $V(\phi)$ corresponds to a certain set of observables $\left(r, n_{s}\right)$, but in general, these parameters are expected to be $O\left(1 / N_{*}\right)$ where $N_{*}$ is the number of e-folds, starting from horizon exit, necessary to solve the standard cosmological problems. In general, this number has a mild dependence on the cosmological history, however under rather reasonable assumptions, $N_{*}$ takes values in the range $N_{*} \simeq 50-60$, that we adopt from now on in our analysis.

Instead of the usual slow-roll parametrisation, one can use a more phenomenological and intuitive way of describing the inflationary phase through its equation of state [20]. During inflation, the equation of state is $p \simeq-\rho \simeq-3 H^{2} M_{P}^{2}$, up to slow-roll corrections, while at the end of inflation $\dot{\phi}^{2} / 2 \simeq V(\phi) \simeq \rho / 2$ and the equation of state is instead $p \simeq 0$. One can thus write that

$$
\frac{p}{\rho}=-1+\frac{\beta}{\left(1+N_{e}\right)^{\alpha}},
$$

where $\alpha$ and $\beta$ are phenomenological parameters and are both positive and of $O(1)$, and $N_{e}$ is the number of remaining e-folds to end inflation $N_{e}(t) \equiv \int_{t}^{t_{e}} d t H$ and it runs from $N_{*}$, at horizon exit, to 0 , when inflation ends. Using energy conservation $\dot{\rho}+3 H(\rho+p)=0$ one gets the following expressions for the tilt and tensor fraction [20]

$$
\begin{aligned}
n_{s}-1 & =-3 \frac{\beta}{\left(N_{*}+1\right)^{\alpha}}-\frac{\alpha}{N_{*}+1} \\
r & =\frac{24 \beta}{\left(N_{*}+1\right)^{\alpha}}
\end{aligned}
$$

The general prediction of the ansatz Eq. (3) is that the tilt is always negative, irrespective of the inflationary

\footnotetext{
$\dagger$ For a nice review of slow-roll inflation see e.g. [21] . Throughout the paper, we will adopt natural units $\hbar=c=1$. As usual, the reduced Planck scale is given by $M_{P}=\left(8 \pi G_{N}\right)^{-1 / 2} \simeq 2.43 \times$ $10^{18} \mathrm{GeV}$.
}

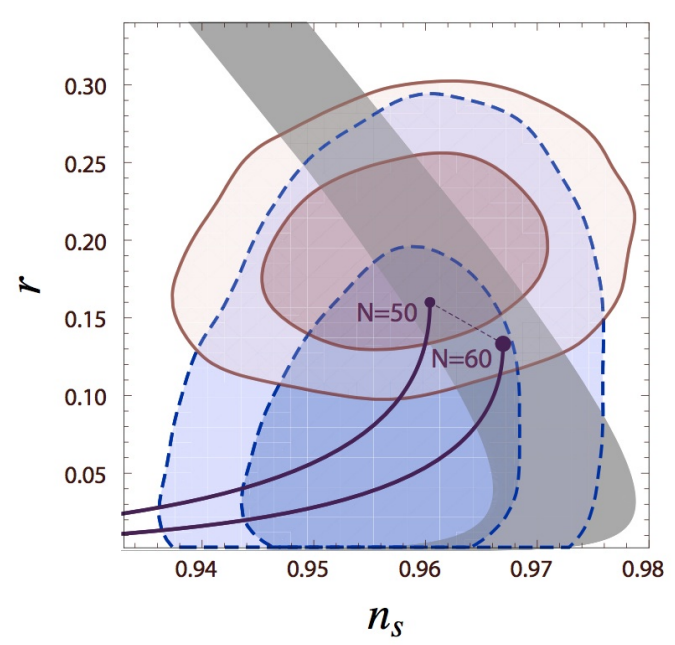

FIG. 1. Confidence regions in the $\left(n_{s}, r\right)$ parameterisation plane. The blue (dashed boundary) areas represent the $68 \%$ and 95\% CL regions of Planck (including a non-zero running), while the red (solid boundary) areas are the $68 \%$ and $95 \%$ $\mathrm{CL}$ regions for BICEP2 only. The grey band represents the predictions of the models captured by the parameterisation Eq. (3) for $50 \leq N_{*} \leq 60$. The solid magenta lines correspond to the natural inflation scenario. For large decay constant $f \gg M_{P}$, they reduce to the $V \propto \phi^{2}$ scenario (short-dashed magenta line).

scenario. In contrast, the value of the tensor-to-scalar ratio can take any value depending on both $\alpha$ and $\beta$. In addition, one can compute the running of the tilt

$$
\alpha_{s} \equiv d n_{s} / d \log k=-\frac{3 \alpha \beta}{\left(1+N_{*}\right)^{\alpha+1}}-\frac{\alpha}{\left(N_{*}+1\right)^{2}},
$$

which is, like the tilt, always negative.

The parameterisation Eq. (3) encodes a variety of models with completely different predictions [20]. Notice however that this phenomenological description of the inflationary phase is not completely equivalent to the slowroll picture, as there is no more freedom in the signs of both the tilt and the running.

From Fig. 1, it is clear that the observationally preferred value of the scalar spectral index $n_{s} \simeq 0.96$ corresponds to two different branches. The first one lies close to the horizontal line $r \approx 0$, in Fig. 1 , and contains for instance Starobinsky models of inflation [22], which are based on the Lagrangian $\sqrt{-g}\left(R+a R^{2}\right)$. In terms of the phenomenological parameterisation Eq. (3), this branch corresponds to $\alpha=2$, and $r \simeq 10^{-2} \beta$. In particular [20], Starobinsky inflation corresponds to $\beta=1 / 2$.

In contrast, the second branch, with significantly higher tensor fraction (appearing as a thick diagonal grey area in Fig. 1) is where chaotic inflation models, $V(\phi) \propto \phi^{n}$ [23], live. In terms of the parameterisation Eq. (3), chaotic scenarios live on the line corresponding to $\alpha=1$. From Eqs. (4), the line in the $\left(n_{s}, r\right)$ plane is given by $n_{s} \simeq 1-\frac{r}{8}$, up to a $O\left(1 / N_{*}\right)$ correction. On 


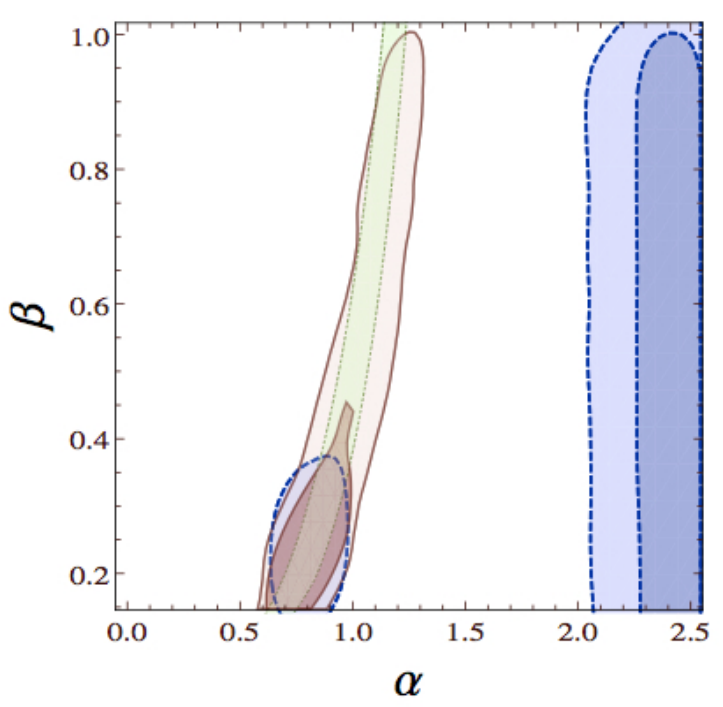

FIG. 2. Confidence regions in the $(\alpha, \beta)$ parameters in Eq. (3). The red areas (solid boundary) represent the $68 \%$ and $95 \%$ CL allowed regions arising from a combined analysis of the Planck, WP and BICEP2 data, while the blue areas (dashed boundary) are the $68 \%$ and $95 \%$ CL allowed regions from the analysis of Planck and WP data. The green region with dotted contours represent the joint $1 \sigma$ preferred region for Planck and BICEP2.

the other hand, the parameter $\beta$ fixes the power of the potential $V \propto \phi^{n}$, as $n=6 \beta$.

The natural inflation scenario [24, 25], in which the inflaton is a pseudo Nambu-Goldstone boson (pNGB), is represented by the purple line in Fig. 1. This scenario, described by the potential $V(\phi) \propto[1-\cos (\phi / f)]$, is captured by the paramterisation Eq. (3) but only for large enough decay constants $f \gtrsim 10 M_{P}$. We recall that in the limit of very large decay constant, $f \gg M_{P}$, Natural inflation reduces to the $\phi^{2}$ scenario represented by the thick purple dots $\left(N_{*}=50\right.$ and $\left.N_{*}=60\right)$ in Fig. 1.

Before describing our cosmological data fits, let us determine the interval spanned by the phenomenological parameters $\alpha$ and $\beta$. First, as explained in Ref. [20], given that inflation ends, i.e. $N_{e} \rightarrow 0$, when $p / \rho \approx 0$, it follows that $\beta$ cannot be much larger that 1 . Second, given that in the most optimistic situation, the tensorto-scalar ratio will be measured at an accuracy of [19] $\Delta r / r=10^{-2}$, it is clear from Eq. (4b) that ${ }^{\ddagger} \alpha \lesssim 2.5$. We

\footnotetext{
¥ A meaningful measurement of the tensor-to-scalar ratio implies that $\Delta r \lesssim r$. Using Eq. (4b), one gets

$$
\alpha \lesssim \log (24 \beta / \Delta r) / \log \left(N_{*}+1\right)
$$
}

which for $\beta \lesssim 1$ and the optimistic percent-level observational error on $r \simeq 0.001$ targeted e.g. by COrE [26] and PIXIE [27] gives $\alpha \lesssim 2.5$. Notice that the above estimate does not change appreciably as it depends only logarithmically on both $N_{*}$ and $\Delta r$.

\begin{tabular}{c|c}
\hline \hline Parameter & Prior \\
\hline$\Omega_{b} h^{2}$ & $0.005 \rightarrow 0.1$ \\
$\Omega_{c} h^{2}$ & $0.001 \rightarrow 0.99$ \\
$\Theta_{s}$ & $0.5 \rightarrow 10$ \\
$\tau$ & $0.01 \rightarrow 0.8$ \\
$\log \left(10^{10} A_{s}\right)$ & $2.7 \rightarrow 4$ \\
$\alpha$ & $0 \rightarrow 2.5$ \\
$\beta$ & $0 \rightarrow 1$ \\
\hline \hline
\end{tabular}

TABLE I. Uniform priors for the cosmological parameters considered here.

shall adopt these priors in our numerical analyses.

The structure of the paper is as follows. In section II, we describe the method followed when performing the fits to the different datasets. Next, in Section III, we present our results in terms of the parameters $\alpha$ and $\beta$ governing the parameterisation Eq. (3), and in terms of the derived, most commonly used inflationary parameters $n_{s}$ and $r$. We also discuss their implications. Finally, we draw our conclusions in Sec. IV.

\section{DATA ANALYSIS}

\section{A. Method}

The phenomenological scenario we explore is described by the following parameter set:

$$
\left\{\omega_{b}, \omega_{c}, \Theta_{s}, \tau, \log \left[10^{10} A_{s}\right], \alpha, \beta\right\},
$$

where $\omega_{b} \equiv \Omega_{b} h^{2}$ and $\omega_{c} \equiv \Omega_{c} h^{2}$ are the physical baryon and cold dark matter energy densities, $\Theta_{s}$ is the ratio between the sound horizon and the angular diameter distance at decoupling, $\tau$ is the reionization optical depth, $A_{s}$ the amplitude of the primordial spectrum and $\alpha$ and $\beta$ are the phenomenological parameters governing the parameterisation given in Eq. (3). In the following, we fix the number of e-folds to $N_{*}=60$. Furthermore, we assume that dark energy is described by a cosmological constant. Table I specifies the priors considered on the cosmological parameters listed above. The commonly used $\left(n_{s}, r\right)$ parameters can be easily obtained using Eqs. (4), however unlike the usual case where the running of the spectral index is a free parameter, the running here is completely fixed through Eq. (5), given $(\alpha, \beta)$ and $N_{*}$. In our analysis, we also consider the socalled inflation consistency relation, relating the tensor spectral index to $r$ through $n_{T}=-r / 8$, which is also valid in this parametrisation ${ }^{\S}$. For our numerical calculations, we use the CAMB Boltzmann code [29], deriv-

\footnotetext{
$\S$ See Eq. (8.125) of [28].
} 


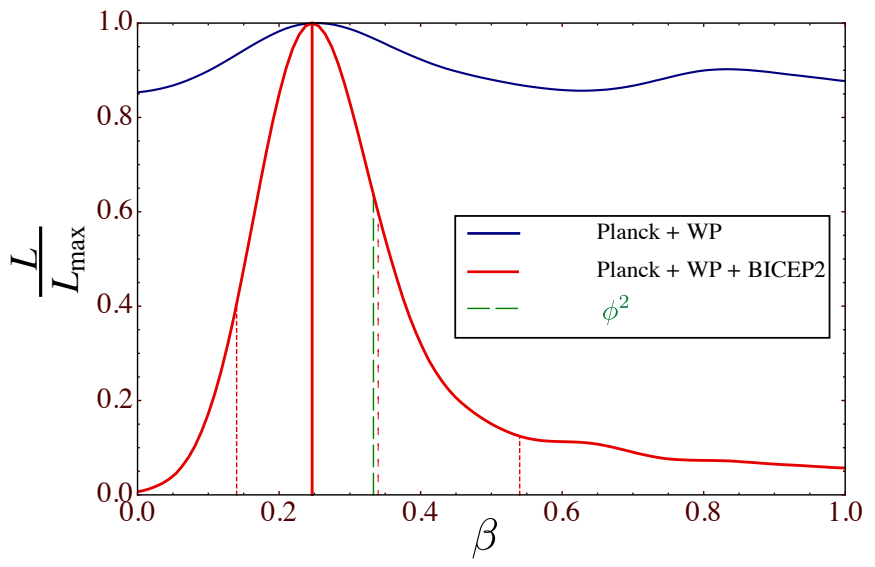

FIG. 3. The derived likelihood distribution for the phenomenological parameter $\beta$ of Eq. (3) using different datasets. The red thick (solid and dashed) vertical lines represent the best-fit ( $\pm 1 \sigma$ intervals) of $\beta$, while the red thin-dashed line stands for the derived mean value of $\beta$, see Table II for details. The quadratic chaotic scenario, corresponding to $\beta=1 / 3$, is represented by a green long-dashed line.

ing posterior distributions for the cosmological parameters from the datasets described in the next section by means of Monte Carlo Markov Chain (MCMC) analyses. Our MCMC results rely on the publicly available MCMC package cosmomc [30] that implements the MetropolisHastings algorithm.

\section{B. Cosmological data}

In our analyses we will consider, as a basic dataset: the Planck CMB temperature anisotropies data [31, 32] together with the 9-year polarization data from the WMAP satellite [33]. The total likelihood for the former data is obtained by means of the Planck collaboration publicly available likelihood code, see Ref. [32] for details. The Planck temperature power spectra extend up to a maximum multipole number $\ell_{\max }=2500$, while the WMAP 9 -year polarization data (WP) is analysed up to a maximum multipole $\ell=23$ [33].

As stated before, very recently, the BICEP2 collaboration has found evidence for the detection of $B$-modes in the multipole range $30<\ell<150$ spanned by their threeyear dataset $[1,2]$, with $6 \sigma$ significance. The detected $B$-mode signal exceeds any known systematics and/or expected foregrounds and is well fitted with a tensor-toscalar ratio $r=0.2_{-0.05}^{+0.07}$. The BICEP2 likelihood has been properly accounted for in our MCMC numerical analyses, by using the latest version of cosmomc.

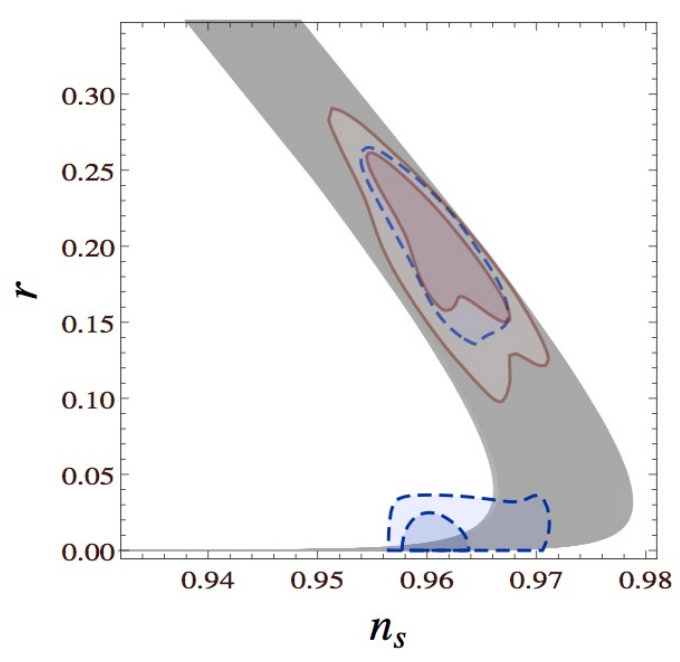

FIG. 4. Confidence regions of the derived parameters $\left(n_{s}, r\right)$ using the parameterisation given by Eq. (3). The colour coding is the same than in Fig. 1. The grey band represents the predictions of the models covered by the parameterisation given by Eq. (3) for $50 \leq N_{*} \leq 60$.

\section{RESULTS}

We represent the results of our MCMC analyses both in the $(\alpha, \beta)$ plane and in the usual $\left(n_{s}, r\right)$ plane. Figure 2 shows the $68 \%$ and $95 \%$ CL contours in $(\alpha, \beta)$. The red solid contours depict the $68 \%$ and $95 \%$ CL allowed regions from the combined analysis of Planck, WP and BICEP2 data, while the blue dashed contours refer to the $68 \%$ and $95 \%$ CL allowed regions from the analysis of Planck and WP data. The green dotted region represents the limits in the $(\alpha, \beta)$ plane inferred from the $1 \sigma$ preferred values for $n_{s}$ and $r$ from Planck and BICEP2 data, respectively. Notice that the results from our MCMC analyses after the combination of Planck, WP and BICEP2 datasets lie precisely within this region. The combination of Planck and WP data is completely insensitive to the $\beta$ parameter, as $\beta$ sets the amount of gravitational waves. The addition of BICEP 2 data, however, strongly constrains the value of $\beta$, as illustrated in Fig. 3, which shows the one-dimensional probability density for the $\beta$ parameter before and after the inclusion of BICEP2 measurements. Figure 3 shows as well the best-fit and the $1 \sigma$ allowed regions for the $\beta$ parameter after considering all the measurements exploited in this study. We also depict in Fig. 3 the value of $\beta$ for the most favoured inflationary scenario, as we shall see in what follows.

Figure 4 depicts the $68 \%$ and $95 \%$ CL allowed contours in the plane of the derived parameters $n_{s}$ and $r$, together with the region covered by the parameterisation given by Eq. (3) for $50 \leq N_{*} \leq 60$. Table II shows the constraints at $68 \%$ confidence level on the cosmological parameters considered in our MCMC analyses for 


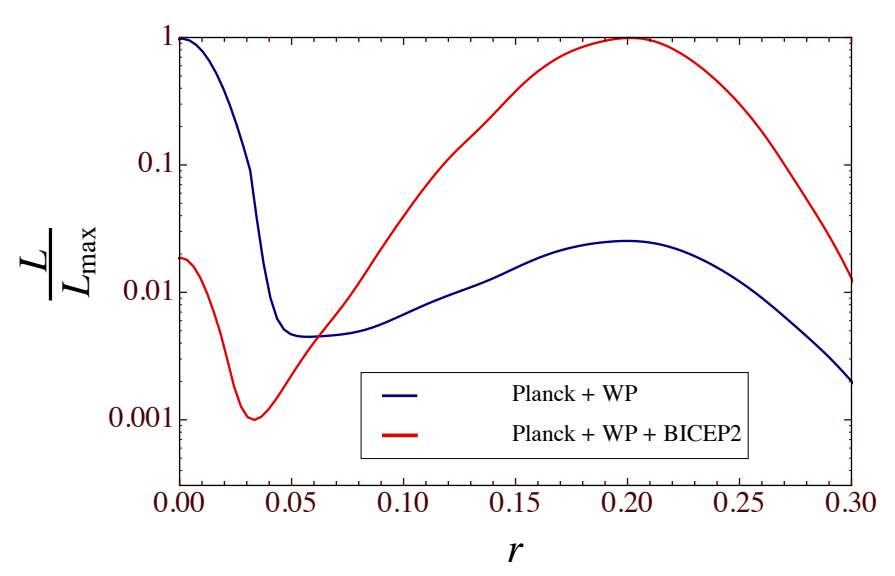

FIG. 5. The derived posterior likelihood distribution for the tensor-to-scalar ratio using different datasets.

the different data combinations explored here. Notice that, when BICEP2 measurements are not considered, $\alpha=2.24 \pm 0.43$ while $\beta=0.50 \pm 0.28$, which corresponds to $n_{s} \simeq 0.96$ and $r<0.18$ at $95 \%$ CL, values that can clearly be inferred from the results depicted in Fig. 4. The constraint we get for Planck and WP data alone is $r<0.18$ at $95 \% \mathrm{CL}$, value to be compared with the value quoted for BICEP2 collaboration for $r=0.16_{-0.05}^{+0.06}[1]$ after subtracting the various foregrounds. Therefore, the upper limits we get on the tensor-to-scalar ratio $r$ from Planck and WP data using the parameterisation given in Eq. (3) are very close to the figure of $r=0.16_{-0.05}^{+0.06}$ reported by the BICEP2 collaboration. This is consistent with the tension found between BICEP2 and Planck using the standard parameters $n_{s}$ and $r$, as the parameterisation used here includes implicitly a non-vanishing running spectral index, see Eq. (5).

The resulting favoured values of $n_{s} \simeq 0.96$ and $r \approx 0$ from the Planck and WP data analysis may be associated to the Starobinsky model of inflation [22]. Indeed, in terms of the phenomenological parameterisation Eq. (3), Starobinsky inflation corresponds to $\alpha=2$ and $\beta=1 / 2[20]$. Another inflationary scenario that can also be identified with these values of $\alpha$ and $\beta$ is Higgs inflation, in which the Standard Model Higgs boson itself is responsible for inflation $[34,35]$. Higgs inflation predicts a scalar spectral index $n_{s} \simeq 0.97$ and a tensor-to-scalar ratio $r \simeq 0.0033$ for $N_{*}=60$ [34] and is indistinguishable observationally from the Starobinsky model.

We can learn from the red contours in Figs. 2 and 4 that, when adding to Planck and WP data the BICEP2 mesurements, models with such large values of $\alpha \sim 2$ are no longer favoured. The resulting mean values of the two parameters are $\alpha=0.88 \pm 0.17$ and $\beta=0.34 \pm 0.20$, which correspond to $n_{s}=0.961 \pm 0.003$ and $r=0.195 \pm 0.037$ (see Table II). This value of $r$ belongs to the second of the branches associated to $n_{s} \simeq 0.96$, which are depicted by the thick diagonal grey area in Figs. 1 and 4 . The one- dimensional posterior probability densities for the derived scalar-to-tensor ratio $r$ are depicted in Fig. 5. Notice that for the two possible data combinations the probability distribution is bimodal, showing two maxima: one is located at $r \simeq 0$ and the other one is located at $r \simeq 0.2$. These two peaks stand for the two possible values of $r$ corresponding to $n_{s} \simeq 0.96$. Each of them is located in one of the two branches shown in Figs. 1 and 4. While the probability distribution function for Planck $+\mathrm{WP}$ data has a global maximum at the $r \simeq 0$ branch, the addition of BICEP2 measurements displaces the global maximum towards the $r \simeq 0.2$ region in the other possible branch. As explained before, it is precisely in this second branch where chaotic inflation models, $V(\phi) \propto \phi^{n}$, live [20]. Chaotic models with quadratic (quartic) potentials predict $n_{s} \simeq 0.96$ and $r \simeq 0.16\left(n_{s} \simeq 0.94\right.$ and $r \simeq 0.32$ ) [20]. Therefore, the mean values of $\alpha$ and $\beta$ resulting from the combined analyses of Planck, WP and BICEP2 data seem to favour $\phi^{2}$ models of chaotic inflation and highly disfavour Starobinsky and Higgs inflation scenarios. The quartic chaotic model also is disfavoured with respect to the quadratic one. The status of the former two inflationary models has also been explored recently in the literature (see e.g. Refs. [36-38]) where it has been found that these two models require either extreme fine-tuning or non-trivial extensions to be compatible with BICEP2 results. Chaotic inflationary models have also been recently revisited in a number of analyses [39-43]. On the other hand, Natural inflation is the only case which can not be analysed in terms of $\alpha$ and $\beta$ except in the large decay constant regime i.e. $f \gg M_{P}$, where $M_{P}$ is the Planck mass. In this case, the constraints are similar to the case of the quadratic chaotic scenario (see Fig. 1). Our derived bound on the tensor-toscalar ratio $(r<0.18$ at $95 \%$ CL) does not put significant constraints on $f$. If on the other hand, we include the BICEP2 datasets, we can translate the $1 \sigma$ interval into a lower bound on the decay constant $f \gtrsim 44.72 M_{P}$ for $N_{*}=50$. This makes Natural inflation practically indistinguishable from the quadratic chaotic scenario, given the present precision. The next generation of observations will improve the situation considerably, allowing for instance to distinguish between the two scenarios if $f \lesssim 30 M_{P}$. See e.g. Refs. [39, 44, 45] for recent appraisals of the Natural inflation scenario. The results previously discussed have been obtained fixing the number of e-folds to $N_{*}=60$. Assuming $N_{*}=50$ instead does not change the main conclusions outlined above.

We conclude this section commenting on the results obtained when using a slightly different upper prior on the $\alpha$ parameter. In general, smaller values of $\alpha$ will give rise to a higher tensor-to-scalar ratio and therefore the tension between Planck and BICEP2 measurements may be alleviated. If we assume an upper prior on $\alpha$ of 2 , the $95 \%$ CL upper bound on the derived tensorto-scalar ratio parameter is slightly larger $(r<0.23$ at $95 \% \mathrm{CL}$ ). On the contrary, when higher values for $\alpha$ are considered, the significance of the tension between 


\begin{tabular}{|c|c|c|}
\hline \hline Parameter & Planck+WP & Planck+WP+BICEP2 \\
\hline$\Omega_{b} h^{2}$ & $0.0209 \pm 0.0002$ & $0.0209 \pm 0.0002$ \\
$\Omega_{c} h^{2}$ & $0.1165 \pm 0.0018$ & $0.1167 \pm 0.0020$ \\
$\theta$ & $1.0409 \pm 0.00055$ & $1.0408 \pm 0.00055$ \\
$\tau$ & $0.086 \pm 0.015$ & $0.078 \pm 0.013$ \\
$\log \left[10^{10} A_{s}\right]$ & $3.063 \pm 0.031$ & $3.047 \pm 0.026$ \\
$\alpha$ & $2.24 \pm 0.43$ & $0.88 \pm 0.17$ \\
$\beta$ & $0.50 \pm 0.28$ & $0.34 \pm 0.20$ \\
$n_{s}$ (derived) & $0.961 \pm 0.002$ & $0.961 \pm 0.003$ \\
$r$ (derived) & $<0.18$ & $0.195 \pm 0.037$ \\
\hline \hline
\end{tabular}

TABLE II. Constraints at $68 \%$ confidence level on cosmological parameters from our analyses for Planck+WP and Planck+WP+BICEP2 data. When quoting upper bounds, we show the $95 \% \mathrm{CL}$ limits. Notice that the scalar spectral index and the tensor to scalar ratio are derived parameters.

Planck and BICEP2 measurements slightly increases, as higher values of $\alpha$ correspond to lower values of $r$. A fit to Planck and WP data gives an upper limit on $r<0.17$ at $95 \%$ CL when using an upper prior on $\alpha$ of 3 . When BICEP2 measurements are included in the analysis, we obtain $n_{s}=0.961 \pm 0.004$ and $r=0.184 \pm 0.040$ $\left(n_{s}=0.961 \pm 0.004\right.$ and $\left.r=0.192 \pm 0.037\right)$ for an upper prior on $\alpha$ of 2 (3). These results are almost identical to the ones quoted in Table II. We have also checked that the posterior probability density profiles for both the parameter $\beta$ and the tensor-to-scalar ratio $r$ do not exhibit a significant prior dependence. Summarising, the effect of the upper prior choice on $\alpha$ barely changes our main results.

\section{CONCLUSIONS}

The recent claimed discovery of primordial gravitational waves by the BICEP2 collaboration has opened a new window into the inflationary paradigm. Chaotic inflation scenarios, highly disfavoured by Planck temperature data, are, after BICEP2 results, among the most plausible ones. Model-independent data analyses are usually presented in terms of the scalar spectral index $n_{s}$ and the tensor-to-scalar ratio $r$, which can then be related to a particular model via the inflationary slowroll parameters. Here we employ an alternative parameterisation due to Mukhanov, describing inflation by an effective equation of state, which captures most of the relevant inflationary scenarios (at least in their basic formulation). Using this parameterisation, one can easily identify the different models as well as derive the usual $n_{s}$ and $r$ parameters. The effective equation of state used here is described by only two parameters, $\alpha$ and $\beta$, since the running of the spectral index $\alpha_{s}$ is no longer a free parameter, as is unambiguously determined once the values of $\alpha$ and $\beta$ are fixed.

Using Markov Chain Monte Carlo methods, we show that the combined analyses of Planck temperature and
WMAP polarisation (WP) data are unable to determine $\beta$, as this last parameter sets the amount of gravitational waves through Eq. (4b). However, these two datasets are able to constrain the other parameter involved, $\alpha$, resulting in a mean value $\alpha=2.24 \pm 0.43$, which corresponds to $n_{s} \simeq 0.96$. Such value of $\alpha$, favoured by the Planck and WP data analyses is associated to both Starobinsky and Higgs inflationary models. The constraint we get on the derived tensor-to-scalar ratio, $r<0.18$ at $95 \% \mathrm{CL}$, is perfectly consistent with the value quoted from the BICEP2 collaboration $\left(r=0.16_{-0.05}^{+0.06}[1]\right)$ after subtracting the various foregrounds. However, this is not necessarily in conflict with the $2 \sigma$ tension found between Planck and BICEP2 measurements when analysing data in terms of the usual $n_{s}$ and $r$ parameters, since the parameterisation used here includes a running which depends exclusively on the $\alpha$ and $\beta$ parameters, as well as on the number of e-folds.

The addition of BICEP2 data to Planck and WP measurements strongly constrains the values of the phenomenological parameters to the values $\beta \simeq 1 / 3$, and $\alpha \simeq 1$. Such values of $\alpha$ correspond to chaotic inflationary models, characterised by a potential $\phi^{n}$, where $n=6 \beta$. Therefore, the results from the combined analysis of Planck, WP and BICEP2 data strongly favour $\phi^{2}$ models of chaotic inflation and rule-out Starobinsky and Higgs inflation scenarios. Upcoming polarisation data from Planck may confirm or falsify the $\phi^{2}$ scenario as the most plausible one for the inflationary period. Future CMB missions, such as COrE [26] and PIXIE [27], combined with galaxy clustering and weak lensing data from the Euclid survey [46] hold the key to establish the amount of primordial $B$-modes and the ensuing theoretical implications, especially if the tensor-to-scalar ratio is as large as suggested by BICEP2. 


\section{ACKNOWLEDGMENTS}

We are grateful to Paolo Creminelli, Scott Dodelson, Carlos Peña Garay and Aaron C. Vincent for their un- valuable comments and suggestions on this work. O.M. is supported by the Consolider Ingenio project CSD200700060, by PROMETEO/2009/116, by the Spanish Ministry Science project FPA2011-29678 and by the ITN Invisibles PITN-GA-2011-289442.
[1] P. A. R. Ade et al. [BICEP2 Collaboration], arXiv:1403.3985 [astro-ph.CO].

[2] P. A. R. Ade et al. [BICEP2 Collaboration], arXiv:1403.4302 [astro-ph.CO].

[3] P. A. R. Ade et al. [Planck Collaboration], arXiv:1303.5076 [astro-ph.CO].

[4] P. A. R. Ade et al. [Planck Collaboration], arXiv:1303.5082 [astro-ph.CO].

[5] C. R. Contaldi, M. Peloso and L. Sorbo, arXiv:1403.4596 [astro-ph.CO].

[6] V. Miranda, W. Hu and P. Adshead, arXiv:1403.5231 [astro-ph.CO].

[7] M. Kawasaki and S. Yokoyama, arXiv:1403.5823 [astroph.CO].

[8] K. N. Abazajian, G. Aslanyan, R. Easther and L. C. Price, arXiv:1403.5922 [astro-ph.CO].

[9] E. Giusarma, E. Di Valentino, M. Lattanzi, A. Melchiorri and O. Mena, arXiv:1403.4852 [astro-ph.CO].

[10] J. -F. Zhang, Y. -H. Li and X. Zhang, arXiv:1403.7028 [astro-ph.CO].

[11] C. Dvorkin, M. Wyman, D. H. Rudd and W. Hu, arXiv:1403.8049 [astro-ph.CO].

[12] M. Archidiacono, N. Fornengo, S. Gariazzo, C. Giunti, S. Hannestad and M. Laveder, arXiv:1404.1794 [astroph.CO].

[13] J. -F. Zhang, Y. -H. Li and X. Zhang, arXiv:1404.3598 [astro-ph.CO].

[14] E. Di Valentino, E. Giusarma, M. Lattanzi, A. Melchiorri and O. Mena, arXiv:1405.1860 [astro-ph.CO].

[15] M. Gerbino, A. Marchini, L. Pagano, L. Salvati, E. Di Valentino and A. Melchiorri, arXiv:1403.5732 [astroph.CO].

[16] C. Cheng and Q. -G. Huang, arXiv:1403.5463 [astroph.CO].

[17] C. Cheng and Q. -G. Huang, arXiv:1405.0349 [astroph.CO].

[18] M. J. Mortonson and U. Seljak, arXiv:1405.5857 [astroph.CO].

[19] S. Dodelson, Phys. Rev. Lett. 112 (2014) 191301 [arXiv:1403.6310 [astro-ph.CO]].

[20] V. Mukhanov, Eur. Phys. J. C 732486 (2013). [arXiv:1303.3925 [astro-ph.CO]].

[21] D. H. Lyth and A. R. Liddle, Cambridge, UK: Cambridge Univ. Press (2009), 497 pp.

[22] A. A. Starobinsky, Phys. Lett. B 9199 (1980).

[23] A. D. Linde, Phys. Lett. B 129, 177 (1983).
[24] K. Freese, J. A. Frieman and A. V. Olinto, Phys. Rev. Lett. 653233 (1990).

[25] F. C. Adams, J. R. Bond, K. Freese, J. A. Frieman and A. V. Olinto, Phys. Rev. D 47426 (1993). [hep$\mathrm{ph} / 9207245]$.

[26] F. R. Bouchet et al. [COrE Collaboration], arXiv:1102.2181 [astro-ph.CO].

[27] A. Kogut, D. J. Fixsen, D. T. Chuss, J. Dotson, E. Dwek, M. Halpern, G. F. Hinshaw and S. M. Meyer et al., JCAP 1107025 (2011). [arXiv:1105.2044 [astro-ph.CO]].

[28] V. Mukhanov, "Physical foundations of cosmology," Cambridge, UK: Univ. Pr. (2005) 421 p.

[29] A. Lewis, A. Challinor and A. Lasenby, Astrophys. J. 538, 473 (2000) [arXiv:astro-ph/9911177].

[30] A. Lewis and S. Bridle, Phys. Rev. D 66, 103511 (2002) [arXiv:astro-ph/0205436].

[31] P. A. R. Ade et al. [Planck Collaboration], arXiv:1303.5062 [astro-ph.CO].

[32] P. A. R. Ade et al. [Planck Collaboration], arXiv:1303.5075 [astro-ph.CO].

[33] C. L. Bennett, D. Larson, J. L. Weiland, N. Jarosik, G. Hinshaw, N. Odegard, K. M. Smith and R. S. Hill et al., arXiv:1212.5225 [astro-ph.CO].

[34] F. L. Bezrukov and M. Shaposhnikov, Phys. Lett. B 659, 703 (2008) [arXiv:0710.3755 [hep-th]].

[35] F. Bezrukov, Class. Quant. Grav. 30, 214001 (2013) [arXiv:1307.0708 [hep-ph]].

[36] S. Ferrara, A. Kehagias and A. Riotto, arXiv:1403.5531 [hep-th].

[37] J. L. Cook, L. M. Krauss, A. J. Long and S. Sabharwal, arXiv:1403.4971 [astro-ph.CO].

[38] K. Nakayama and F. Takahashi, arXiv:1403.4132 [hep$\mathrm{ph}]$.

[39] P. Creminelli, D. López Nacir, M. Simonović, G. Trevisan and M. Zaldarriaga, arXiv:1404.1065 [astro-ph.CO].

[40] N. Okada, V. N. Şenoğuz and Q. Shafi, arXiv:1403.6403 [hep-ph].

[41] P. Di Bari, S. F. King, C. Luhn, A. Merle and A. SchmidtMay, arXiv:1404.0009 [hep-ph].

[42] N. Kaloper and A. Lawrence, arXiv:1404.2912 [hep-th].

[43] P. Creminelli, D. López Nacir, M. Simonović, G. Trevisan and M. Zaldarriaga, arXiv:1405.6264 [astro-ph.CO].

[44] K. Freese and W. H. Kinney, arXiv:1403.5277 [astroph.CO].

[45] K. Kohri, C. S. Lim and C. -M. Lin, arXiv:1405.0772 [hep-ph].

[46] L. Amendola et al. [Euclid Theory Working Group Collaboration], Living Rev. Rel. 16, 6 (2013) [arXiv:1206.1225 [astro-ph.CO]]. 